\title{
Motor Phenotype in Neurodegenerative Disorders: Gait and Balance Platform Study Design Protocol for the Ontario Neurodegenerative Research Initiative (ONDRI)
}

Manuel Montero-Odasso ${ }^{\mathrm{a}, \mathrm{b}, \mathrm{c}, *}$, Frederico Pieruccini-Faria ${ }^{\mathrm{a}, \mathrm{c}}$, Robert Bartha $^{\mathrm{k}}$, Sandra E. Black ${ }^{\mathrm{e}, \mathrm{f}}$, Elizabeth Finger ${ }^{\mathrm{g}}$, Morris Freedman ${ }^{\mathrm{h}}$, Barry Greenberg ${ }^{\mathrm{i}}$, David A. Grimes ${ }^{\mathrm{j}}$, Robert A. Hegele ${ }^{\mathrm{d}, \mathrm{k}}$, Christopher Hudson ${ }^{1}$, Peter W. Kleinstiver ${ }^{\mathrm{m}}$, Anthony E. Lang ${ }^{\mathrm{n}}$, Mario Masellis ${ }^{\mathrm{e}}$, Paula M. McLaughlin ${ }^{\mathrm{m}}$, Douglas P. Munoz ${ }^{\mathrm{o}}$, Stephen Strother ${ }^{\mathrm{p}}$, Richard H. Swartz ${ }^{\mathrm{q}}$, Sean Symons ${ }^{\mathrm{r}}$, Maria Carmela Tartaglia ${ }^{\mathrm{s}}$, Lorne Zinman ${ }^{\mathrm{e}}$, Michael J. Strong ${ }^{\mathrm{k}, \mathrm{h}}$ ONDRI Investigators and William McIlroy $\mathrm{f}, \mathrm{n}, \mathrm{t}$

${ }^{a}$ Department of Medicine, Division of Geriatric Medicine, Parkwood Hospital, University of Western Ontario, London, ON, Canada

${ }^{\mathrm{b}}$ Department of Epidemiology and Biostatistics, University of Western Ontario, London, ON, Canada ${ }^{\mathrm{c}}$ Lawson Health Research Institute, London, ON, Canada

${ }^{\mathrm{d}}$ Department of Biochemistry, Schulich School of Medicine and Dentistry, University of Western Ontario, London, ON, Canada

${ }^{\mathrm{e}}$ Department of Medicine, Division of Neurology, Sunnybrook Health Sciences Centre, University of Toronto, ON, Canada

${ }_{\mathrm{f}}^{\mathrm{f}}$ Canadian Partnership for Stroke Recovery Sunnybrook Site, Sunnybrook Health Sciences Centre, University of Toronto, Toronto, ON, Canada

${ }^{\mathrm{g}}$ Department of Clinical Neurological Sciences, Schulich School of Medicine and Dentistry, University of Western Ontario, London, ON, Canada

${ }^{\mathrm{h}}$ Department of Medicine (Neurology), Baycrest Health Sciences and University of Toronto, Toronto, ON, Canada; Rotman Research Institute, Baycrest Centre for Geriatric Care, Toronto, ON, Canada

${ }^{\mathrm{i}}$ Toronto Dementia Research Alliance, University Health Network, Toronto, ON, Canada

${ }^{\mathrm{j}}$ Department of Medicine, The Ottawa Hospital, Faculty of Medicine, University of Ottawa, Ottawa, ON, Canada

${ }^{\mathrm{k}}$ Department of Medical Biophysics, Schulich School of Medicine and Dentistry, Robarts Research Institute, University of Western Ontario, London, ON, Canada

${ }^{1}$ School of Optometry and Vision Science, University of Waterloo, Waterloo, ON, Canada

${ }^{\mathrm{m}}$ Schulich School of Medicine and Dentistry, University of Western Ontario, London, ON, Canada

${ }^{\mathrm{n}}$ Morton and Gloria Shulman Movement Disorders Clinic and the Edmond J. Safra Program in Parkinson's Disease, Toronto Western Hospital and the Department of Medicine, University of Toronto, ON, Canada

${ }^{\circ}$ Centre for Neuroscience Studies, Queen's University, Kingston, ON, Canada

${ }^{\mathrm{p}}$ Department of Medical Biophysics, Rotman Research Institute, Baycrest, University of Toronto, ON, Canada q Sunnybrook Health Sciences Centre, University of Toronto, Stroke Research Program, Toronto, ON, Canada

\footnotetext{
*Correspondence to: Manuel Montero-Odasso, $\mathrm{MD}, \mathrm{PhD}$, FRCPC, Department of Medicine, Division of Geriatric Medicine,
} 
${ }^{\mathrm{r}}$ Department of Medical Imaging, Sunnybrook Health Sciences Centre, Toronto, ON, Canada

${ }^{\mathrm{s}}$ Department of Medicine and Division of Neurology, University of Toronto, Tanz Centre for Research in Neurodegenerative Diseases, University of Toronto, ON, Canada

${ }^{\mathrm{t}}$ Department of Kinesiology, University of Waterloo, Waterloo, ON, Canada

Accepted 16 May 2017

\begin{abstract}
.
Background: The association of cognitive and motor impairments in Alzheimer's disease and other neurodegenerative diseases is thought to be related to damage in the common brain networks shared by cognitive and cortical motor control processes. These common brain networks play a pivotal role in selecting movements and postural synergies that meet an individual's needs. Pathology in this "highest level" of motor control produces abnormalities of gait and posture referred to as highest-level gait disorders. Impairments in cognition and mobility, including falls, are present in almost all neurodegenerative diseases, suggesting common mechanisms that still need to be unraveled.

Objective: To identify motor-cognitive profiles across neurodegenerative diseases in a large cohort of patients.

Methods: Cohort study that includes up to 500 participants, followed every year for three years, across five neurodegenerative disease groups: Alzheimer's disease/mild cognitive impairment, frontotemporal degeneration, vascular cognitive impairment, amyotrophic lateral sclerosis, and Parkinson's disease. Gait and balance will be assessed using accelerometers and electronic walkways, evaluated at different levels of cognitive and sensory complexity, using the dual-task paradigm.

Results: Comparison of cognitive and motor performances across neurodegenerative groups will allow the identification of motor-cognitive phenotypes through the standardized evaluation of gait and balance characteristics.

Conclusions: As part of the Ontario Neurodegenerative Research Initiative (ONDRI), the gait and balance platform aims to identify motor-cognitive profiles across neurodegenerative diseases. Gait assessment, particularly while dual-tasking, will help dissect the cognitive and motor contribution in mobility and cognitive decline, progression to dementia syndromes, and future adverse outcomes including falls and mortality.
\end{abstract}

Keywords: Alzheimer's disease, amyotrophic lateral sclerosis, balance, dementia, dual-tasking, frontotemporal dementia, gait, neurodegeneration, Parkinson's disease, vascular cognitive impairment

\section{INTRODUCTION}

\section{Cognitive and motor decline coexist in neurodegenerative disorders}

An important goal of medicine is to reduce the gap between life expectancy and disability-free life expectancy. A substantial portion of this gap is related to cognitive impairment and mobility limitations, which ultimately manifest as dementia, falls, and ambulation loss. Interestingly, these manifestations often coexist in elderly people: falling is a common geriatric syndrome affecting about a third of older adults each year, and dementia syndromes affect $8 \%$ of older adults over the age of 65 , ascending to $35 \%$ in those over age 85 [1-3]. Until recently, falls and dementia were studied as distinct syndromes, which contributed to gaps in understanding the cognitivemotor interactions that affect pathways to disability in older populations [4]. Epidemiological evidence shows that the coexistence of cognitive and motor impairments in older adults, particularly gait slowing, is an early phenomenon in the trajectory to cognitive decline and dementia [5-8]. The association of cognitive and motor impairments in aging and neurodegenerative diseases, is not merely additive due to their high prevalence alone; rather, emerging evidence for neuroimaging studies support that gait regulation and cognitive processes share common brain regions and networks [5, 9-12]. These common brain networks include the cortico-basal ganglia-thalamocortical connections, which play a pivotal role in selecting movements and postural synergies that meet an individual's needs and environmental constraints. Pathology in this "highest level" of motor control produces abnormalities of gait and postural control that are collectively referred to as "highest level gait disorders" [13, 14]. The cerebral cortex, basal ganglia, thalamus, and subcortical white matter are the critical sites of pathology in dementing diseases and as such, most patients with the highest level gait disorders have some degree of cognitive impairment and vice versa. Specifically, recent studies have shown that a good performance in executive functioning is essential for planning and monitoring goal-directed behavior, including walking gait $[15,16]$. This evidence suggests that especially in individuals with neurodegeneration, further 
investigation on the relationship between cognitive and gait impairments could ultimately help better elucidate the underlying processes in these shared brain networks.

Although a clinical hallmark of vascular cognitive impairment (VCI), Alzheimer's disease (AD), mild cognitive impairment (MCI), and frontotemporal dementia (FTD) is cognitive decline, motor impairments are often present at different stages of these diseases. Motor impairment symptoms include bradykinesia, extrapyramidal rigidity, spasticity, and gait disorders, such as cautious gait or gait slowing [17, 18]. Similarly, Parkinson's disease (PD) and amyotrophic lateral sclerosis (ALS) initially show pure motor symptoms, but in both diseases, severity of gait and balance impairments are often associated with worse cognitive functioning. Motor disorders, and specifically gait disorders, may be present at an early stage of dementia syndromes, as demonstrated in large population studies, including the Bronx Aging Study [19] and the Sydney Older Persons Study [20]. When focusing on populations at risk for dementia syndromes, such as older adults with MCI, we and others have found early coexistence of specific cognitive deficiencies (executive dysfunction, working memory deficits, and attention) and gait abnormalities, particularly while dual-tasking [21, 22]. This evidence suggests that there is a transition period whereby cognitive loss occurred concurrently with slowing of gait.

The prospective assessment of quantitative gait and balance variables in the targeted neurodegenerative diseases included in the Ontario Neurodegenerative Disease Research Initiative (ONDRI) will expand our understanding about the gait, balance, and cognition relationships, helping to target interventions to reduce the risk of falls in these disorders and to delay the associated disability. Moreover, this will provide deeper insights regarding which gait/balance assessments specifically can be most efficiently used for diagnostic purposes and for monitoring responses to pharmacological and non-pharmacological treatment in neurodegenerative diseases [9, 23-25].

\section{Cognitive and motor decline in \\ neurodegenerative disorders may have modifiable common risk factors}

Understanding gait and cognitive dysfunction as a pathology affecting common brain networks may also unravel the role of modifiable factors including vascular damage, chronic inflammation, or yet to be defined factors, in addition to the neurodegenerative process [26]. For instance, vascular risk factors accelerate cognitive decline and are associated with future development of $\mathrm{AD}$ and vascular dementia [27]. Among the different cognitive domains, executive function and cognitive speed processing are most likely to be altered by the presence of vascular risk factors, specifically hypertension, as demonstrated in the Canadian Study of Health and Aging [28]. Similarly, "The Gait and Brain Study" showed that older adults with MCI who scored high in a vascular risk factors composite score, including atrial fibrillation, hypercholesterolemia, smoking, previous myocardial infarction, and hypertension, were more likely to present slowing gait, greater dual-task cost on gait, depressed mood, and executive dysfunction $[29,30]$. Imaging studies have revealed that prefrontal and frontal atrophy and white matter hyperintensities (WMH) are associated with slow gait velocity, balance, falls, and future disability in community older adults [31]. Anatomically, the close proximity of frontal subcortical networks that control both motor and cognitive functions may explain why frontal atrophy and WMH may simultaneously cause dysfunction in both systems. Because of their watershed vascularization, frontal-temporal neuronal networks are highly susceptible to vascular risk factors, brain microvascular disease, and blood oxygenation levels. As shown in other organs, the accumulation of microvascular ischemic changes in the brain may also contribute to the motor and cognitive decline seen in neurodegenerative processes $[15,30]$.

From a mechanistic perspective, it is difficult to know the exact nature of the interaction between cognitive function and gait impairments in older adults with neurodegenerative disorders due to the coexistence of cognitive and motor problems found in some of these diseases, including ALS and PD. An experimental strategy to investigate these mechanisms is to compare individuals with different neurodegenerative processes that also affect different neural systems (e.g., cognitive, sensory, motor, and sensorimotor). The use of a dual-task paradigm, performing a cognitive task while walking, may help characterize the nature of gait deficits by increasing the "stress" on cognitive networks that regulate gait control $[5,21$, 22]. Following the principle that with the presence of sensorimotor impairments, gait control is increasingly reliant on more conscious cognitive processes, impairment in gait, and balance control may result from progressive cognitive decline in neurodegenerative disorders. 


\section{The role of cognition in gait: The dual-task} paradigm and gait variability

Although walking has long been considered primarily as an automatic motor task, emerging evidence suggests that this view is overly simplistic [32]. Cognitive function may play a key role even in the regulation of routine walking, particularly in older adults. This functioning may also be linked, at least in part, to the challenges in dynamic balance control, which is required during routine walking and relies on brain networks that are instrumental to executive cognitive control [32].

Disturbances to the control of gait and balance, including increased falls occurrence, are common consequences of nearly all neurological disorders. In both healthy and neurologically-impaired individuals, there is considerable evidence for the interaction between control of balance (standing and walking) and cognitive capacity, especially performance in attention and executive functioning. The most compelling line of evidence comes from dual-task studies on gait and balance, when the ability to perform concurrent executive functions can be significantly limited during tasks with increasing challenges to balance control (e.g., walking) [33]. Such dual-task studies also reveal that the cognitive demands of dynamic balance (e.g., walking) increase in the face of background neurologic disorder [34]. In other words, gait performance while dual-tasking can be seen as an important way to understand cognitive capacity and brain function [5].

Attention is a necessary cognitive resource for maintaining normal walking and there is evidence that cognitive and attention deficits are independently associated with postural instability, impairment in performing daily living activities, and future falls [33, 35]. The role of cognition in walking is even more marked in people with neurodegenerative disorders and cognitive dysfunction, whose gait performance is affected by any extra attentional load. Since the seminal "stops walking when talking" study [34] demonstrated that the inability to maintain a conversation while walking is a marker for future falls in older adults. Observing people walking while they perform a secondary task ("dual-task paradigm") has become the accepted way to assess the interaction between cognition, gait, and risk of mobility decline and falling. Previous research on the effect of dual-tasking on gait performance showed specific associations between gait slowing, executive dysfunction and attention deficits [32, 36-43].
In a previous study in MCI and early AD populations, our group has demonstrated that impairments in several cognitive domains (attention, executive function, and working and semantic memory) are associated with both slow single task gait velocity and also slower gait velocity under dual-task conditions, showing that these specific cognitive domains are crucial for maintaining normal gait performance [44]. Impairment in gait while dual-tasking has also been previously described in PD [45-50], FTD [51-53], and ALS [18] populations.

A sensitive measure of dynamic stability during walking is gait variability, defined as the stride-tostride variation in time [54]. This measure quantifies gait automaticity, with greater variability indicating reduced consistency of steps cycles and a more unstable gait pattern. Evaluating gait variability is an accurate methodology to identify subtle changes in walking due to pathological conditions or disease. For instance, cognitively normal older adults have low gait variability; however, high gait variability has been described in PD and AD, and has been associated with high risk of future falls and mobility decline [55]. Additionally, previous studies have demonstrated that gait variability may serve as a clinically relevant parameter in the evaluation of mobility and may be a responsive measure for different interventions in fall prevention [56]. Although both ALS and FTD show higher gait variability and falls, there are no studies investigating the relationship between them. However, the lack of research using the motor phenotype of these diseases to predict adverse events and disease prognosis may be explained by the low prevalence of both ALS and FTD.

\section{Overall rationale}

To our knowledge, this collaborative approach to research spanning the most common neurodegenerative diseases and including vascular causes, has never been undertaken in the same project. The current research is also developing standardized assessment protocols to evaluate gait and mobility across disease states, which allows for comparisons and enables identification of both common and unique factors across these disorders. Furthermore, necessary and more comprehensive gait and balance assessments will be created, based on these evaluations of brain health in each neurodegenerative disease state. This tool is not currently available, but would be very valuable for risk prediction and pre-emptive management across care silos and eventually in primary practice. 
Current therapeutic approaches in neurodegenerative diseases tend to be directed toward single biological mechanisms, which may be inadequate given the complexity of these multifaceted diseases. Through this integrated discovery approach, we have the unique opportunity to identify multiple motor markers of brain health that will contribute to the development of clinical biomarkers for neurodegenerative disease. This may ultimately be relevant for the identification of subjects in pre-symptomatic stages and for personalized pharmacological or therapeutic approaches that may have better effectiveness than approaches presently utilized. Hence, this wellstructured and integrated longitudinal study has the potential to deliver significant impacts on health care in the rapidly growing area of neurodegenerative disease.

\section{Main research questions for the gait and balance platform}

Our longitudinal design aims to answer important questions that will help clinicians and basic scientists to improve knowledge about diagnosis and prognosis of neurodegenerative disorders and their underlying mechanisms. Some of these questions are: Are there identifiable "motor-cognitive" profiles for the different neurodegenerative disorders of interest? Can selected "motor-cognitive" profiles serve as clinical biomarkers of disease progression as well as fall risk? Which is the neuro-anatomical substrate (brain local atrophy, white matter disease, etc.) of the "motor-cognitive" profiles identified? What are the associations between potential modifiable risk factors for the "motor-cognitive" profiles identified?

Specifically, based on recently described motorcognitive profiles in individuals with different MCI [9], we will characterize and compare cognitive and gait interactions in the five cohort groups recruited to diagnose, monitor, and assess the effect of treatments.

\section{Specific aims}

Aim 1: To identify motor-cognitive profiles based on gait and balance performance in five neurodegenerative diseases.

We hypothesize that neurodegenerative diseases with accelerated cognitive worsening in attention and executive functions will show more gait and balance impairments at baseline.

Aim 2: To test the predictive ability of the motor-cognitive profiles identified to predict neurodegenerative progression.
We hypothesize that gait parameters will help to predict disease progression including cognitive and neurological aspects.

Aim 3: To investigate the neuro-anatomical correlates of the motor-cognitive profiles identified in the five neurodegenerative diseases of interest.

We hypothesize that the five neurodegenerative diseases will share neuro-anatomical characteristics in grey matter and white matter structural changes and that these common characteristics will correlate with motor-cognitive profiles.

\section{METHODOLOGY}

This protocol follows guidelines for observational studies along with the Strengthening the Reporting of Observational Studies in Epidemiology (STROBE) checklist for cohort studies [57].

\section{Design}

The overall design of ONDRI and details on participant characteristics have been previously reported in detail elsewhere [58]. In brief, up to 500 participants who have one of the following diseases: AD/MCI (120:60 AD; 60 MCI); ALS (40); FTD (50); PD (140); or VCI (150) are being enrolled into this longitudinal study from 13 recruitment centers throughout Ontario, Canada. Figure 1 outlines the workflow of the study and key project research questions. Inclusion and exclusion criteria for the study as a whole is delineated below. Ethics approval was obtained in all participating institutions.

\section{Inclusion/Exclusion criteria}

Participants must meet each of the following general criteria for enrolment into the study.

\section{General inclusion}

1) Written informed consent must be obtained and documented using the site's approved Letter of Information and Consent Form.

2) Participant must have self-reported levels of proficiency in English for speaking and understanding spoken language, and score 7 out of 10 or higher on the two Language Experience and Proficiency Questionnaire (LEAP-Q) questions.

3) Participant must have $\geq 8$ years of education.

4) Participant must have a minimum Montreal Cognitive Assessment (MoCA) [59] score 
The Ontario Neurodegenerative Disease Research Initiative Workflow

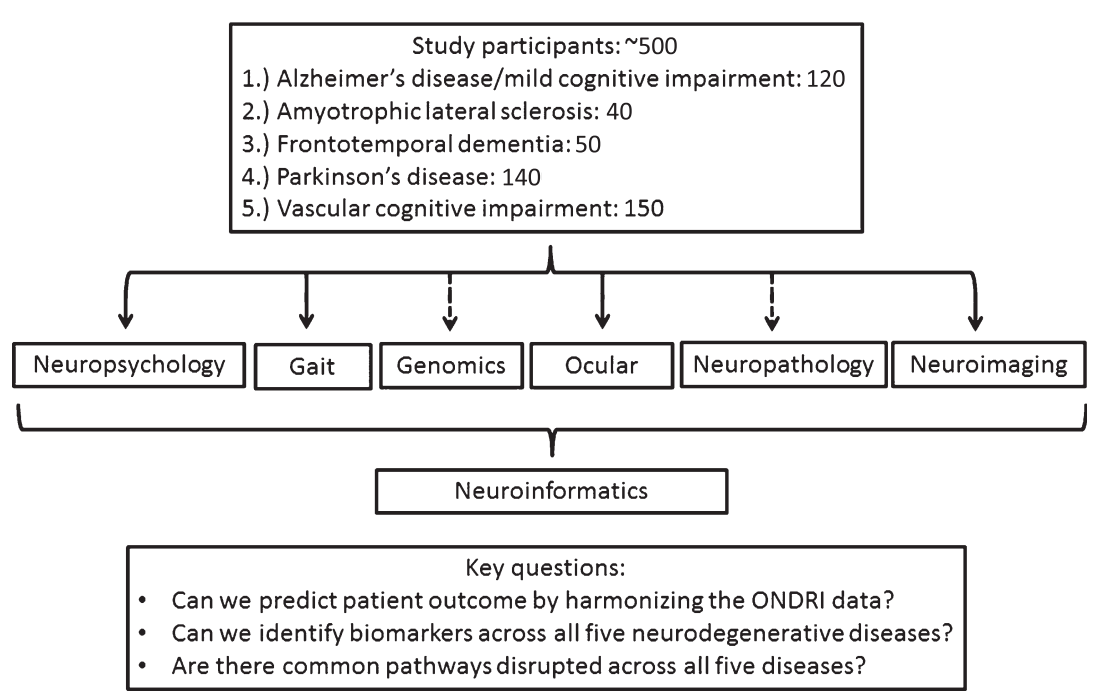

Fig. 1. The Ontario Neurodegenerative Disease Research Initiative workflow. Dashed arrows represent single time assessments.

of $\geq 18$ (except for FTD and Atypical AD in whom score must be $>14$ ).

5) Participant must have a reliable Study Partner. The Study Partner must: (a) Interact regularly with the participant (i.e., have contact with the participant at least once a month over the phone, email, or face-to-face); (b) Know the participant well enough to answer questions about the her/his cognitive abilities, communication skills, mood, and daily functioning (i.e., have known the participant for at least two years); (c) Provide written informed consent and complete study questionnaires; (d) Be able to assist in compliance with study procedures (if required).

6) Participant must have geographic accessibility to the study site.

\section{General exclusion}

1) Serious underlying disease other than the disease being studied, which in the opinion of the investigator may interfere with the participant's ability to participate fully in the study.

2) Any disease that would/could lead to death over the next three to five years (i.e., cardiac/renal/liver cancer) with poor prognosis.

3) History of alcohol or drug abuse, which in the opinion of the investigator, may interfere with the participant's ability to comply with the study procedures.
4) Presence of any of the following clinical conditions: Substance abuse within the past year; Unstable cardiac, pulmonary, renal, hepatic, endocrine, hematologic, or active malignancy or infectious disease; AIDS or AIDS-related complex; Unstable psychiatric illness defined as psychosis (hallucinations or delusions), lifelong history of major depression, or untreated late- onset major depression within 90 days of the screening visit.

5) Being currently enrolled in a disease modifying therapeutic trial.

These criteria will be verified through a standardized clinical, neurological, and musculoskeletal examination by the Principal Investigator. Specific criteria for inclusion and exclusion in each cohort of disorders has been previously detailed [58].

\section{Follow-up}

All participants involved in the study will undergo established and standardized assessments including: neuroimaging, neuropsychology, genomics, ocular (eye movements and retinal imaging), and gait and balance assessments. These assessments will be repeated every 12 months after the baseline assessment during the three years of follow-up (and every six months for the ALS cohort). Phone interviews are also occurring every 6 months between yearly assessments. 


\section{Platforms assessments}

The ONDRI study consists of six different platforms: neuroimaging, neuropsychology, neuropathology, genomics, ocular (eye movements and retinal imaging), and gait and balance assessments. Each platform includes its own set of assessments, and the present protocol focuses on the "Gait and Balance Platform", having the rest of the platforms assessments been described elsewhere [58]. Table 1 summarizes the assessments and variables collected in each time point that will aid in the identification of motor-cognitive profiles. In brief, each participant will have baseline genomics, standardized comprehensive annual magnetic resonance imaging, detailed cognitive, speech and language evaluation, retinal imaging, and eye tracking, as well as measures of gait and balance performance.

In this protocol, we focus on the uniqueness and main objectives of the "Gait and Balance Platform" and we provide additional details below regarding the neurocognitive and the imaging platform, as it will assist in the motor-cognitive profile identification.

In the ONDRI Neuropsychology platform, the following neuropsychological domains are being evaluated: attention, executive, memory, speech production, language, and visuospatial function, with a particular focus on cognitive domains that reflect frontal network functioning, including complex attention, executive cognition, and social cognition. Testing used to assess for these cognitive domains are described in Table 2. Finally, questionnaires that provide measures of neuropsychiatric functioning, meta-cognitive skills, personality, and activities of daily living are administered. From the ONDRI Neuroimaging platform, variables related to microvascular brain disease and WMH burden are being evaluated. WMH severity will be characterized using the Fazekas scale on brain images [60]. This four-point qualitative scale provides a measurement of the severity of WMH and is reported separately for the peri-ventricular white matter and the deep

Table 1

Characteristics of all variables used in the protocol

\begin{tabular}{|c|c|c|c|c|c|}
\hline Type & Variable name & Form & Source & Time (follow-ups) & $\begin{array}{c}\text { Standardized } \\
\text { tool }\end{array}$ \\
\hline \multirow[t]{8}{*}{ Demographic } & Age & Continuous & Questionnaire & $\mathrm{BL}$ & NA \\
\hline & Sex & Dichotomous & Questionnaire & BL & NA \\
\hline & Height & Continuous & Questionnaire & $\mathrm{BL}, \mathrm{Y} 1, \mathrm{Y} 2, \mathrm{Y} 3$ & NA \\
\hline & Weight & Continuous & Questionnaire & $\mathrm{BL}, \mathrm{Y} 1, \mathrm{Y} 2, \mathrm{Y} 3$ & NA \\
\hline & Body mass index & Continuous & Questionnaire & $\mathrm{BL}, \mathrm{Y} 1, \mathrm{Y} 2, \mathrm{Y} 3$ & NA \\
\hline & Diagnosis & Nominal & Questionnaire & $\mathrm{BL}, \mathrm{Y} 1, \mathrm{Y} 2, \mathrm{Y} 3$ & NA \\
\hline & Cognitive status (e.g., dementia) & Dichotomous & Questionnaire & $\mathrm{BL}, \mathrm{Y} 1, \mathrm{Y} 2, \mathrm{Y} 3$ & NA \\
\hline & Education $(\mathrm{y})$ & Continuous & Questionnaire & $\mathrm{BL}$ & NA \\
\hline \multirow{5}{*}{$\begin{array}{l}\text { Outcomes } \\
\text { Primary and secondary }\end{array}$} & Gait velocity (primary) & Continuous & Mobility assessment & $\mathrm{BL}, \mathrm{Y} 1, \mathrm{Y} 2, \mathrm{Y} 3$ & Yes \\
\hline & Gait variability (primary) & Continuous & Mobility assessment & $\mathrm{BL}, \mathrm{Y} 1, \mathrm{Y} 2, \mathrm{Y} 3$ & Yes \\
\hline & Dual-task cost (secondary) & Continuous & Mobility assessment & $\mathrm{BL}, \mathrm{Y} 1, \mathrm{Y} 2, \mathrm{Y} 3$ & Yes \\
\hline & $\begin{array}{l}\text { Postural sway area (secondary) } \\
\text { - Transition and standing }\end{array}$ & Continuous & Mobility assessment & $\mathrm{BL}, \mathrm{Y} 1, \mathrm{Y} 2, \mathrm{Y} 3$ & Yes \\
\hline & $\begin{array}{l}\text { Postural sway velocity (secondary) } \\
\text { - Transition and standing }\end{array}$ & Continuous & Mobility assessment & $\mathrm{BL}, \mathrm{Y} 1, \mathrm{Y} 2, \mathrm{Y} 3$ & Yes \\
\hline \multirow[t]{9}{*}{ Covariates } & Fear of falling & Dichotomous & Questionnaire & $\mathrm{BL}, \mathrm{Y} 1, \mathrm{Y} 2, \mathrm{Y} 3$ & Yes \\
\hline & Balance confidence scale & Ordinal & Questionnaire & $\mathrm{BL}, \mathrm{Y} 1, \mathrm{Y} 2, \mathrm{Y} 3$ & Yes \\
\hline & History of Falls & $0,1,2,3+$ & Questionnaire & $\mathrm{BL}, \mathrm{Y} 1, \mathrm{Y} 2, \mathrm{Y} 3$ & Yes \\
\hline & Activities of daily living & Ordinal, 0-16 & Questionnaire & $\mathrm{BL}, \mathrm{Y} 1, \mathrm{Y} 2, \mathrm{Y} 3$ & Yes \\
\hline & Assistive device use & Dichotomous & Questionnaire & $\mathrm{BL}, \mathrm{Y} 1, \mathrm{Y} 2, \mathrm{Y} 3$ & Yes \\
\hline & Psychiatric disorders & Dichotomous & Questionnaire & $\mathrm{BL}, \mathrm{Y} 1, \mathrm{Y} 2, \mathrm{Y} 3$ & Yes \\
\hline & Comorbidities & Dichotomous & Questionnaire & $\mathrm{BL}, \mathrm{Y} 1, \mathrm{Y} 2, \mathrm{Y} 3$ & NA \\
\hline & Cardiovascular Factors & Ordinal & Questionnaire & $\mathrm{BL}$ & Yes \\
\hline & Chronic Medications & Dichotomous & Questionnaire & $\mathrm{BL}, \mathrm{Y} 1, \mathrm{Y} 2, \mathrm{Y} 3$ & NA \\
\hline \multirow[t]{5}{*}{ Descriptives } & ApoE4 (from genomics platform) & Dichotomous & Blood work & $\mathrm{BL}, \mathrm{Y} 1, \mathrm{Y} 2, \mathrm{Y} 3$ & Yes \\
\hline & $\begin{array}{l}\text { White matter lesions and volumetric } \\
\text { analysis (from imaging platform) }\end{array}$ & Continuous & 3T MRI scan & $\mathrm{BL}, \mathrm{Y} 1, \mathrm{Y} 2, \mathrm{Y} 3$ & Yes \\
\hline & Death & Dichotomous & Medical record & $\mathrm{BL}, \mathrm{Y} 1, \mathrm{Y} 2, \mathrm{Y} 3$ & NA \\
\hline & Conversion to disease & Dichotomous & Questionnaire & $\mathrm{BL}, \mathrm{Y} 1, \mathrm{Y} 2, \mathrm{Y} 3$ & NA \\
\hline & Instrumental Activities of daily living & Scale & Questionnaire & $\mathrm{BL}, \mathrm{Y} 1, \mathrm{Y} 2, \mathrm{Y} 3$ & Yes \\
\hline
\end{tabular}

BL, baseline; Y, year (1,2,3); NA, not available; 3T MRI, 3 Tesla magnetic resonance imaging. 
Table 2

Cognitive domains evaluated across diseases and tests used in the Neuropsychology Platform protocol

\begin{tabular}{|c|c|c|c|c|}
\hline Variable name & Modality & Source & Time (follow-ups**) & $\begin{array}{c}\text { Standardized } \\
\text { tool }\end{array}$ \\
\hline Sensory acuity & Auditory screen; vision screen & Audiometry Visuometry & $\mathrm{BL}, \mathrm{Y} 1, \mathrm{Y} 2, \mathrm{Y} 3$ & Yes \\
\hline $\begin{array}{l}\text { Estimated intellectual } \\
\text { functioning }\end{array}$ & WASI-II: vocabulary and matrix reasoning & Pencil and paper test & $\mathrm{BL}, \mathrm{Y} 1, \mathrm{Y} 2, \mathrm{Y} 3$ & Yes \\
\hline $\begin{array}{l}\text { Attention, working memory, } \\
\text { and Processing Speed }\end{array}$ & WAIS-III: digit span; SDMT & Pencil and paper test & $\mathrm{BL}, \mathrm{Y} 1, \mathrm{Y} 2, \mathrm{Y} 3$ & Yes \\
\hline $\begin{array}{l}\text { Complex attention and } \\
\text { Executive functioning }\end{array}$ & $\begin{array}{l}\text { DKEFS: verbal fluency; Trail Making Test; } \\
\text { WASI-II: matrix reasoning; DKEFS: } \\
\text { color-word interference }\end{array}$ & Pencil and paper test & $\mathrm{BL}, \mathrm{Y} 1, \mathrm{Y} 2, \mathrm{Y} 3$ & Yes \\
\hline Theory of mind & ECAS: judgment of preference* & Pencil and paper test & $\mathrm{BL}, \mathrm{Y} 1, \mathrm{Y} 2, \mathrm{Y} 3$ & Yes \\
\hline $\begin{array}{l}\text { Visuoperceptual and } \\
\text { construction }\end{array}$ & $\begin{array}{l}\text { VOSP: incomplete letters; JLO; BVMT-R } \\
\text { copy; }\end{array}$ & Pencil and paper test & $\mathrm{BL}, \mathrm{Y} 1, \mathrm{Y} 2, \mathrm{Y} 3$ & Yes \\
\hline $\begin{array}{l}\text { Speech production, language, } \\
\text { and discourse }\end{array}$ & $\begin{array}{l}\text { Leap-Q Modified; BDAE: semantic probe; } \\
\text { sentence intelligibility }{ }^{\dagger} \text {; diadochokinetic } \\
\text { task }^{\dagger} \text {; maximum sustained phonation } \\
\text { task }^{\dagger} \text {; BNT; TAWF: verb naming; DKEFS: } \\
\text { verbal fluency; BDAE: cookie theft picture } \\
\text { description; procedural discourse task; } \\
\text { sequenced story task }\end{array}$ & Pencil and paper test & $\mathrm{BL}, \mathrm{Y} 1, \mathrm{Y} 2, \mathrm{Y} 3$ & Yes \\
\hline Memory & $\begin{array}{l}\text { RAVLT; BVMT-R }{ }^{\dagger \dagger} \text {; face/name association; } \\
\text { SDMT: symbol-digit recall }\end{array}$ & Pencil and paper test & $\mathrm{BL}, \mathrm{Y} 1, \mathrm{Y} 2, \mathrm{Y} 3$ & Yes \\
\hline $\begin{array}{l}\text { Neuropsychiatric, } \\
\text { metacognition, personality, } \\
\text { and functional }\end{array}$ & $\begin{array}{l}\text { NPI-Q-informant; IRI-self and -informant; } \\
\text { Short IQ-code-self and -informant; } \\
\text { mini-SAM; iADL scale - informant; } \\
\text { physical self-maintenance } \\
\text { scale-informant; RSMS-informant: Social } \\
\text { norms questionnaire; IAS-B5-informant; } \\
\text { BIS/BAS-informant }\end{array}$ & Questionnaires & $\mathrm{BL}, \mathrm{Y} 1, \mathrm{Y} 2, \mathrm{Y} 3$ & Yes \\
\hline
\end{tabular}

BDAE, Boston diagnostic aphasia examination; BIS/BAS, behavioral inhibition system/behavioral activation system; BNT, Boston naming test; BVMT-R, brief visuospatial memory test-revised; DKEFS, Delis-Kaplan executive function system; ECAS, Edinburgh Cognitive and Behavioral ASL Screen; IAS, interpersonal adjective scales; IRI, interpersonal reactivity index; JLO, judgment of line orientation; NPI-Q, neuropsychiatric inventory questionnaire; RAVLT, Rey auditory verbal learning task; RSMS, revised self-monitoring scale; SAM, survey of autobiographical memory; SDMT, symbol-digit modalities test; TAWF - test of adolescent/adult word finding; VOSP, visual object and space perception battery; WAIS-III, Wechsler Adult Intelligence Scale, 3rd edition; WASI, Wechsler abbreviated scale of intelligence, 2nd edition. * Only given to the ALS and FTD cohorts. ${ }^{\dagger}$ Not given to the AD/MCI cohort. ${ }^{\dagger \dagger}$ Not given to the ALS cohort. ${ }^{* *}$ NOTE: The ALS cohort completes the Neuropsychology Platform assessment every 6 months.

white matter. WMH will also be assessed quantitatively with volumetric measures by using the Lesion Explorer software following the Canadian Dementia Imaging Protocol (http://www.cdip-pcid.ca.) developed by co-authors Drs. Robert Bartha, Sandra Black, and others. These volumes are further parcellated into 26 brain regions using a semi-automated brain extraction (SABRE) [61].

\section{Measurements and procedures for the gait balance platform assessments}

Quantitative gait parameters will be assessed using wearable inertial sensors (Gulf Coast Inc.; Shimmer Inc.) worn bilaterally on the ankles and at the hip (Fig. 2). Acceleration data will be used to calculate foot-contact and foot-off times to determine step and stride timing data. Overall gait velocity is determined by timing the standard walk distance. In two sites, gait performance will be additionally assessed using electronic walkway systems (GAITRite ${ }^{\circledR}$ or PKMas ${ }^{\circledR}$ ), which automatically determines spatiotemporal gait parameters from imbedded sensors activated by foot pressure. A computer processes the footsteps, providing data for both spatial and temporal parameters. Our team has established excellent correlation $(r=0.9)$ and reliability for gait measures retrieved using electronic walkways and accelerometers.

\section{Gait assessment protocol}

For walking conditions, all individuals will perform walks along a 6-meter path while wearing hip and ankle worn accelerometers. Individuals will perform three main walking blocks: 1) preferred walking speed; 2) dual-task walking; and 3) fast walking. In the case that a participant uses an assistive aid (cane, 

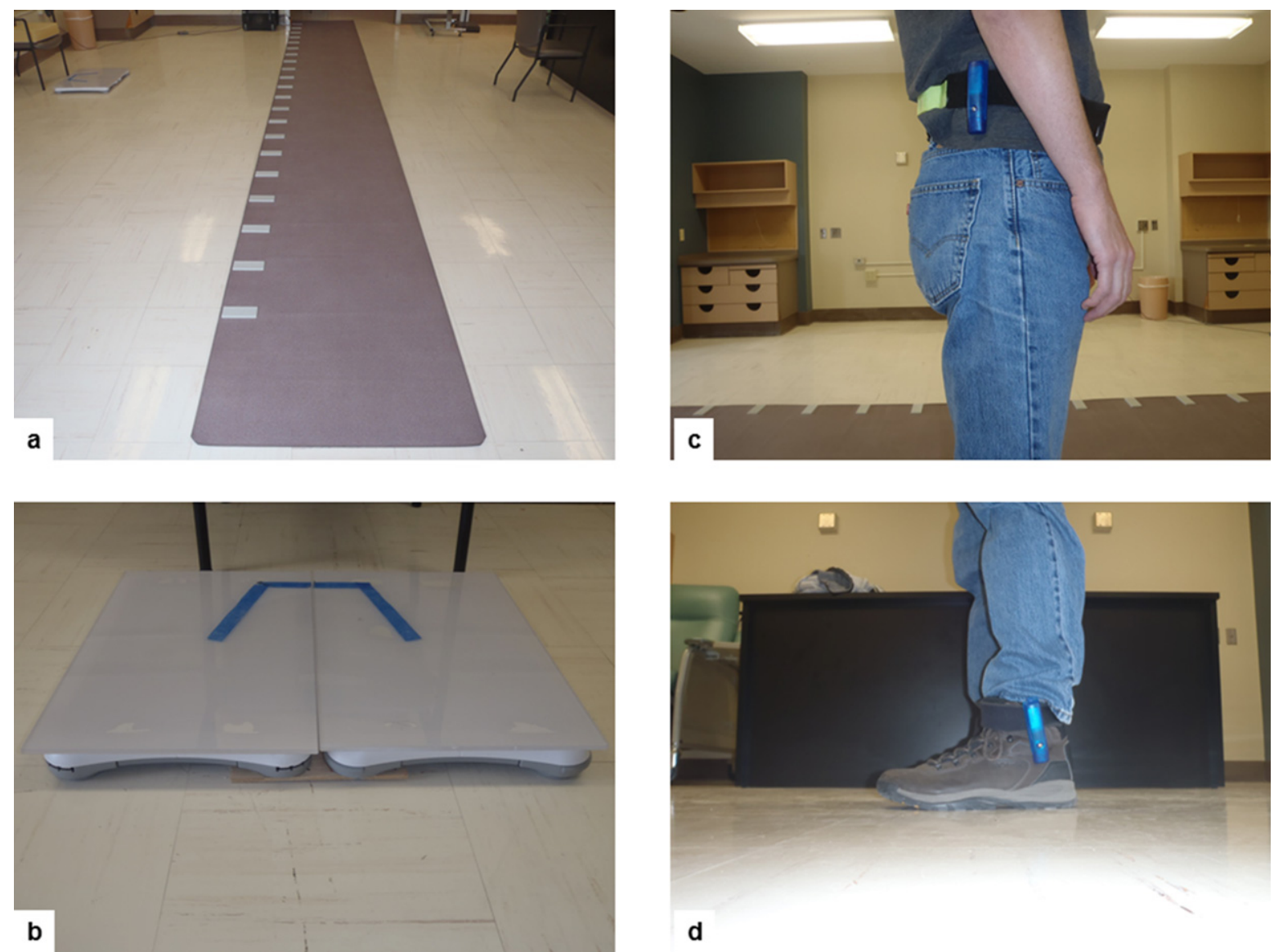

Fig. 2. Equipment that will be used to assess gait and balance performance: (a) GaitRITE ${ }^{\circledR}$ mat for gait assessments; (b) Wii board adapted to assess balance control during rest conditions and sit-to-stand transitions; (c) Accelerometers attached to hips and ankles will be used in sites where they are available.

walker), the tester will determine the participant's capacity to walk safely without the use of the aid. With the willingness of the participant, they will then perform one test walk without the use of the aid. If the participant is able to safely walk without the use of the assistive aid, all subsequent testing will be performed without its use. In all walks, participants will start one meter before the beginning of the 6-meter pathway and continue to travel one meter past the end of the platform. Distance is marked in the pathway with tapes. This procedure is in place to ensure steady-state walking and to minimize any effects of acceleration and de-acceleration during the course of the walk.

The first walking block will measure self-selected or preferred walking, called "single-task gait". Participants will be instructed to "walk at a comfortable and secure pace". For the preferred walking speed during "single-task gait", a total of three walks will be performed. Participants with slow walking speed, less than $0.6 \mathrm{~m} / \mathrm{s}$, or participants with lower limb disability, will be allowed to complete one walk if they are not able to perform the three trials. The second walking block will be dual-task walking and includes three separate walks. The first dual-task administered will have the participant walk while simultaneously counting backward by serial $1 \mathrm{~s}$ from 100 (i.e., 100$99-98$... ) out loud. The second dual-task will have the participant repeat the walk but this time, generating animal names (semantic fluency test) out loud. In the third dual-task, the participant will be asked to walk the platform while subtracting $7 \mathrm{~s}$ from 100 (i.e., 100-93-86 ... ) out loud. During this trial, participants are encouraged to keep walking even if they cannot do the subtractions. These dual-task conditions selected are based on previous research which demonstrated that counting backwards requires both working memory and attention [62]. For these tests, 
participants will be instructed to pay attention to both gait and cognitive task; if a participant stops either task during the trial, they will be prompted to continue. Not instructing to prioritize gait over cognitive task or vice-versa allows both gait and cognitive task to vary. This has previously been shown to provide a better representation of what happens naturally and better reflects activity of daily living situations [63]. The evaluator will record any counting errors during walking. The third and last walking block will include fast walking and the participants will be instructed to walk as fast and safe as they can without running.

\section{Static balance assessment}

Balance assessments will be conducted while standing on portable force boards (Wii, Nintendo Inc.) to capture the center of pressure $(\mathrm{CoP})$. Measures of CoP displacement variability (RMS) in the mediolateral and anterior posterior planes are calculated along with stance symmetry, sway path length, sway area, and mean velocity [34]. Additional measures of the estimate of center of mass will be calculated from the acceleration of the hip worn accelerometer. Participants are instructed to stand still with their arms crossed for $30 \mathrm{~s}$ for each of the following conditions: 1) eyes open/normal stance; 2) eyes closed/normal stance; 3) eyes open/narrow stance (standard Romberg); and 4) eyes closed/narrow stance (standard Romberg). For normal stance, a standardized position will be adopted from the participants' normally occurring stance: $17 \mathrm{~cm}$ between heel centers with an angle of $14^{\circ}$ between the long axes of the foot. For the narrow stance condition, participants' feet will be positioned together. The tasks are performed in the listed order as they reflect an increase in task challenge. Participants who are unable to perform the eyes open standard stance or eyes closed standard stance will not attempt the narrow stance conditions.

\section{Transitions assessment}

Vertical transitions will be evaluated using a single sit-to-stand procedure. For this, participants are instructed to stand from a seated position and remain standing as still as possible for $20 \mathrm{~s}$. Feet are in a preferred position with respect to the seat pan. Participants are first instructed to perform the sit-to-stand in a "preferred" manner (use the arms of the chair or use hand support on their thighs). If capable, individuals should attempt a second trial without the use of their arms (crossed in front). Data collected from the portable force boards and from body worn accelerom- eters will characterize the movement and stability control $(\mathrm{CoP})$ after standing so that the dynamic and re-stabilization phases are captured. These measures will provide accurate information about postural stability during real-world situations involving complex postural transitions.

\section{Balance confidence scale}

Balance confidence will be evaluated using the Activities-Specific Balance Confidence Scale [64]. Respondents self-rate their confidence in their balance with a 16-item questionnaire about a series of daily tasks. The described tasks range in difficulty from those of basic daily living (e.g., walking around the house, going up and down stairs) to more difficult tasks generally performed in the community (e.g., walking in crowded areas like shopping centers, using escalators). Respondents are asked to rate their confidence on a scale from $0 \%$ (no confidence) to $100 \%$ (complete confidence) based on the following cue question: "How confident are you that you will not lose your balance or become unsteady when you...". The scale's wide range of item difficulty makes it well-suited to assessing balance as a construct in populations with varying levels of functioning, including high-functioning community living seniors. This scale has been validated in previous studies as a marker of risk of falling $[64,65]$.

\section{Falls}

A fall is defined as "unintentionally coming to rest on the ground, floor, or other lower level and not due to a seizure, syncope, or an acute stroke" [66]. Typically, events caused by overwhelming environmental hazards (e.g., being struck by a moving object) are not considered a fall. Recurrent falls are defined as 'two or more events in a 12-month period during follow up'. Falls will be recorded using a validated questionnaire at each time point face-to-face assessment (annually) and every 6 months using phone interviews during the three years of follow-up. This methodology has been validated and used in our previous research and others $[67,68]$.

\section{Gait and balance outcome measures}

The motor (gait and balance) outcome measures below are used to search for differences across the five clinical groups in order to identify the motorcognitive profiles. 


\section{Gait outcomes}

Gait velocity and the coefficient of variation $(\mathrm{CoV})$ of stride time are the primary gait outcome measures. The coefficient of variation is calculated according to the following formula: $\mathrm{CoV}=[$ (standard deviation $/$ mean $) \times 100$ ]. Dual-task gait cost $($ DTC) will be calculated using the following formula: [(single-task gait value- dual-task gait value) / single-task gait value] $\times 100$ and is expressed in percentage. DTC quantifies the magnitude of the effect of cognitive load on motor performance (e.g., velocity and variability).

\section{Balance outcomes}

These outcome measures will include anteriorposterior plane and medium-lateral plane sway displacements and total sway area of the CoP. Displacements of the body in frontal and sagittal direction will be recorded using a force platform. In addition, the sway area will be calculated by multiplying the frontal diameter with the sagittal diameter. Measures of RMS in the medio-lateral and anterior posterior planes are calculated along with stance symmetry, sway path length, sway area, and mean velocity and used as additional secondary outcomes.

\section{Analysis}

\section{Baseline characteristics}

Gait and balance parameters will be descriptively summarized using either means and standard deviations or frequencies and percentages, as appropriate. Comparisons of the changes in the mean balance confidence score will be assessed using One-way analysis of variance (ANOVA) with covariates when necessary. Post-hoc comparisons across diagnostic groups will be conducted using Tukey's HSD test when appropriate. The role of small vessels diseases in motor-cognitive phenotypes will be evaluated by weighting the burden of WMH in VCI and $\mathrm{AD} / \mathrm{MCI}$ using quantities measure of such as $\mathrm{WMH}$ volume.

\section{Cross-sectional analysis}

Comparisons among disease groups will be made using appropriate multivariable regression models based on the nature of outcomes, adjusting for other baseline characteristics. The $\mathrm{CoV}$ for stride time is used to quantify gait variability under each testing condition. A two-way repeated measures ANOVA will be performed to evaluate the effect of cognitive status based on performance in global cognition (MoCA) [59] and detailed neuropsychological testing across the increasing complexity of gait tasks (dual-task conditions) and their interaction (group $\times$ condition). If the overall F-test was significant, post hoc testing will be performed (Tukey's test) to identify which pair-wise comparisons between groups are significantly different to one another. Adjustments will be performed for age, sex, number of comorbidities, and history of falls. The level of statistical significance is set at $p<0.05$ (twosided).

\section{Longitudinal analysis}

The relationship between gait and balance quantitative variables and further cognitive decline (in all the groups) and incident dementia (in groups free of dementia syndromes at baseline) will be analyzed using mixed models to account for the correlations in repeated responses in the same subject. Standard procedures will be followed to build appropriate models prior to interpretation of results. Falls incidence will also be analyzed during follow-up. Time to falling will be first analyzed using unadjusted survival curves between those with low and high gait variability at baseline, using the non-parametric Kaplan-Meier technique $[69,70]$. This will be followed by fitting Cox proportional hazards regression models. This will allow the refinement of the Kaplan-Meier estimates by adjusting for baseline $\mathrm{CoV}$ as a continuous covariate, as well as other baseline covariates (e.g., age, sex, ApoE4, educational level, depressive symptoms, neuropsychological measures). Repeated falls will be analyzed as recurrent events using a marginal Cox proportional hazards model [70, 71]. The Cox proportional hazards models will be tested for the proportional hazards assumption, which, if found to be violated, will be followed by the addition of time-dependent covariates collected at the follow-up assessments. In addition, we will be also analyzing annual prospective risks using logbinomial regression models [72]. Logistic regression models will be used to evaluate whether cognition particularly executive function and attention are associated with increasing gait variability over time.

Two-sided $p<0.05$ will be considered statistically significant. The statistical significance of the results will be adjusted by Hochberg's variation of the Bonferroni procedure for multiple testing [73]. All calculations will be performed using SPSS software package version 21.1 (SPSS Inc., Chicago, IL). 


\section{DISCUSSION}

To our knowledge, this is one of the first attempts to have a collaborative, multimodality approach in exploring the critical link between motor and cognitive behavior across five common neurodegenerative conditions. This cohort study has the potential to reveal evidence based disease-specific motor-cognitive profiles that may serve to inform new diagnostic and/or assessment protocols to track disease progression and/or recovery. Given the growing recognition of the importance of mobility, gait, and balance on overall health and function, the focus on encompassing a range of neurodegenerative diseases in this project will assist in the development of standardized mobility assessment protocols allowing comparisons across disease states. While gait may well serve as the 6th vital sign [74-77] and a motor biomarker for neurodegenerative diseases [25], a standard approach used to measure and challenge gait (e.g., dual-tasking) in clinical settings is critically important to advance the use of this index in routine care. This study will serve as a step toward a more unified and quantitative approach for the assessment of mobility in clinical settings while evaluating patients with cognitive impairments or at risk of dementia [25].

Mechanistically, this cohort study will help to understand the basis of the co-existence of motor and cognitive impairments in neurodegeneration. Specifically, how motor-cognitive interactions modulate the risk of future adverse outcomes, including falls and fractures, and the progression to further cognitive decline and dementia syndromes, as we schematized in Fig. 3. Established neuroanatomical changes in neurodegeneration includes focal and generalized brain atrophy, $\mathrm{WMH}$, and cortical and lacunar cerebral infarcts, Lewy bodies, neuritic plaques, and neurofibrillary tangles. These pathology changes are very common in the five neurodegenerative disorders studied in the ONDRI cohort and may affect shared brain cortical areas and networks that regulate motor-cognitive processes (Fig. 3).

In addition, this study will explore person-specific determinants of mobility that will contribute to development of 1) motor clinical biomarkers for neurodegenerative diseases that may ultimately be relevant toward the identification of pre-symptomatic individuals, particularly brain small vessel disease; and 2) personalized treatments based on motor and motor-cognitive phenotypes to reduce further mobility disability and falls. Multiple motor biomarkers or phenotypes identified may vary depending on the stage of the disorder being treated, the genetic predispositions discovered to be contributing to an individual's disease, and the possible role of mixed pathologies. Hence, this well-structured and integrated longitudinal cohort study has the potential to contribute with a significant impact to health care in the rapidly growing area of neurodegenerative diseases.

A distinctive aspect of this platform in the ONDRI study is the use of reliable and valid instruments to assess mobility quantitatively in clinical settings including use of new cost-effective, wearable technologies. The growing wave of wearable technologies has been led by consumer grade tools that provide relatively little direct clinical utility. Incorporating the

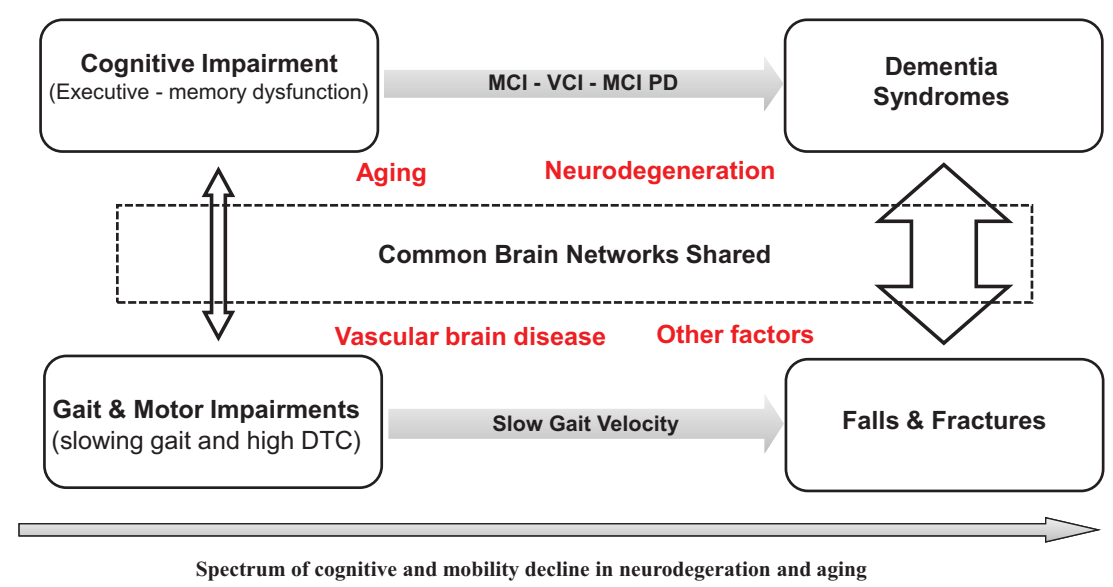

Fig. 3. Potential mechanism affecting the common brain structures and networks that regulate gait control and cognitive performance. Adapted from Montero-Odasso et al. [5]. 
current sensor technology into clinically meaningful user interfaces and outcomes will help to transform such use into standard clinical care. The opportunity to extract such meaningful metrics about gait and balance as well as motor-cognitive interactions (e.g., stride variability and dual-task gait cost) from wearable inertial sensors has been well demonstrated in research models. The current study will adapt such developments for the core measures of gait and balance with the goal to develop standardized clinical tool-kits based on the gait and balance measures used in this protocol. Gait variability is an emerging measure of gait stability that provides insight into the cortical control of gait. Our gait protocol includes three walks for single-gait to assure that more than 12 steps are recorded in order to obtain a reliable measure of gait variability while single walking [78].

In summary, despite the known overlap in neural regions and networks for both cognitive processes and motor performance, still the underlying common and unique mechanisms of the motor-cognitive interactions and their mediators and moderators in neurodegenerative disorders are unknown. This information is crucial for the further understanding of how neurodegenerative diseases evolve to cognitive and mobility disabilities and to identify potential underlying reversible factors. This will facilitate future diagnoses and ameliorate their prognoses and treatments.

\section{ACKNOWLEDGMENTS}

Our gratitude goes to Alanna Black and Yanina Sarquis-Adamson from the "Gait and Brain Lab" for their assistance editing the final version of the manuscript. This research is conducted with the support of the Ontario Brain Institute, an independent non-profit corporation, funded partially by the Ontario government. The opinions, results and conclusions are those of the authors and no endorsement by the Ontario Brain Institute is intended or should be inferred. We also thank the financial support from the Temerty Family Foundation.

The ONDRI Gait and Balance Platform is led by Drs. McIlroy and Montero-Odasso. We thank and acknowledge the consent and cooperation of all ONDRI participants. Many thanks to the ONDRI investigators (lead investigator: Dr. Michael Strong) and the ONDRI governing committees: executive committee; steering committee; publication committee; recruiting clinicians; assessment platforms leaders; and the ONDRI project management team. For a full list of the ONDRI investigators, please visit: http://www.ONDRI.ca/people.

Dr. Freedman received support from the Saul A. Silverman Family Foundation as a part of Canada International Scientific Exchange Program project and Morris Kerzner Memorial Fund.

Authors' disclosures available online (http://j-alz. com/manuscript-disclosures/17-0149r1).

\section{REFERENCES}

[1] (1994) Canadian study of health and aging: Study methods and prevalence of dementia. CMAJ 150, 899-913.

[2] (2006) Third Canadian Consensus Conference on Diagnosis and Treatment of Dementia. Available from: http://wwwccc dtdca/cccdtd/home2html.

[3] Thomas VS, Darvesh S, MacKnight C, Rockwood K (2001) Estimating the prevalence of dementia in elderly people: A comparison of the Canadian Study of Health and Aging and National Population Health Survey approaches. Int Psychogeriatr 13 Supp 1, 169-175.

[4] Montero-Odasso M, Bherer L, Studenski S, Gopaul K, Oteng-Amoako A, Woolmore-Goodwin S, Stoole P, Wells J, Doherty T, Zecevic AA, Galinsky D, Rylett RJ, Jutai J, Muir-Hunter S, Speechley M, Camicioli R (2015) Mobility and Cognition in Seniors. Report from the 2008 Institute of Aging (CIHR) Mobility and Cognition Workshop. Can Geriatr J 18, 159-167.

[5] Montero-Odasso M, Verghese J, Beauchet O, Hausdorff JM (2012) Gait and cognition: A complementary approach to understanding brain function and the risk of falling. $\mathrm{J} \mathrm{Am}$ Geriatr Soc 60, 2127-2136.

[6] Boyle PA, Buchman AS, Wilson RS, Leurgans SE, Bennett DA (2009) Association of muscle strength with the risk of Alzheimer disease and the rate of cognitive decline in community-dwelling older persons. Arch Neurol 66, 13391344.

[7] Fitzpatrick AL, Buchanan CK, Nahin RL, DeKosky ST, Atkinson HH, Carlson MC, Williamson JD (2007) Associations of gait speed and other measures of physical function with cognition in a healthy cohort of elderly persons. J Gerontol A Biol Sci Med Sci 62, 1244-1251.

[8] Soumare A, Tavernier B, Alperovitch A, Tzourio C, Elbaz A (2009) A cross-sectional and longitudinal study of the relationship between walking speed and cognitive function in community-dwelling elderly people. J Gerontol A Biol Sci Med Sci 64, 1058-1065.

[9] Montero-Odasso M, Oteng-Amoako A, Speechley M, Gopaul K, Beauchet O, Annweiler C, Muir-Hunter SW (2014) The motor signature of mild cognitive impairment: Results from the gait and brain study. J Gerontol A Biol Sci Med Sci 69, 1415-1421.

[10] Rosso AL, Studenski SA, Chen WG, Aizenstein HJ, Alexander NB, Bennett DA, Black SE, Camicioli R, Carlson MC, Ferrucci L, Guralnik JM, Hausdorff JM, Kaye J, Launer LJ, Lipsitz LA, Verghese J, Rosano C (2013) Aging, the central nervous system, and mobility. J Gerontol A Biol Sci Med Sci 68, 1379-1386.

[11] Annweiler C, Beauchet O, Bartha R, Wells JL, Borrie MJ, Hachinski V, Montero-Odasso M (2013) Motor cortex and gait in mild cognitive impairment: A magnetic resonance 
spectroscopy and volumetric imaging study. Brain 136, 859871.

[12] Annweiler C, Beauchet O, Bartha R, Montero-Odasso M (2013) Slow gait in MCI is associated with ventricular enlargement: Results from the Gait and Brain Study. J Neural Transm 120, 1083-1092.

[13] Alexander NB (1996) Gait disorders in older adults. $J$ Am Geriatr Soc 44, 434-451.

[14] Nutt JG, Marsden CD, Thompson PD (1993) Human walking and higher-level gait disorders, particularly in the elderly. Neurology 43, 268-279.

[15] Montero-Odasso M, Hachinski V (2014) Preludes to brain failure: Executive dysfunction and gait disturbances. Neurol Sci 35, 601-604.

[16] Sorond FA, Cruz-Almeida Y, Clark DJ, Viswanathan A, Scherzer CR, De JP, Csiszar A, Laurienti PJ, Hausdorff JM, Chen WG, Ferrucci L, Rosano C, Studenski SA, Black SE, Lipsitz LA (2015) Aging, the central nervous system, and mobility in older adults: Neural mechanisms of mobility impairment. J Gerontol A Biol Sci Med Sci 70, 1526-1532.

[17] Ricciardi L, Bloem BR, Snijders AH, Daniele A, Quaranta D, Bentivoglio AR, Fasano A (2014) Freezing of gait in Parkinson's disease: The paradoxical interplay between gait and cognition. Parkinsonism Relat Disord 20, 824-829.

[18] Radovanovic S, Milicev M, Peric S, Basta I, Kostic V, Stevic $Z$ (2014) Gait in amyotrophic lateral sclerosis: Is gait pattern differently affected in spinal and bulbar onset of the disease during dual task walking? Amyotroph Lateral Scler Frontotemporal Degener 15, 488-493.

[19] Verghese J, Levalley A, Hall CB, Katz MJ, Ambrose AF, Lipton RB (2006) Epidemiology of gait disorders in community-residing older adults. J Am Geriatr Soc 54, 255261.

[20] Waite LM, Grayson DA, Piguet O, Creasey H, Bennett HP, Broe GA (2005) Gait slowing as a predictor of incident dementia: 6-year longitudinal data from the Sydney Older Persons Study. J Neurol Sci 229-230, 89-93.

[21] Montero-Odasso M, Bergman H, Phillips NA, Wong CH, Sourial N, Chertkow H (2009) Dual-tasking and Gait in people with mild cognitive impairment. The effect of working memory. BMC Geriatr $\mathbf{9}, 41$.

[22] Montero-Odasso M, Muir SW, Speechley M (2012) Dualtask complexity affects gait in people with mild cognitive impairment: The interplay between gait variability, dual tasking, and risk of falls. Arch Phys Med Rehabil 93, 293299.

[23] Montero-Odasso M, Wells JL, Borrie MJ, Speechley M (2009) Can cognitive enhancers reduce the risk of falls in older people with mild cognitive impairment? A protocol for a randomised controlled double blind trial. BMC Neurol 9, 42 .

[24] Montero-Odasso M, Muir-Hunter SW, Oteng-Amoako A, Gopaul K, Islam A, Borrie M, Wells J, Speechley M (2015) Donepezil improves gait performance in older adults with mild Alzheimer's disease: A phase II clinical trial. J Alzheimers Dis 43, 193-199.

[25] Montero-Odasso M (2016) Gait as a biomarker of cognitive impairment and dementia syndromes. Quo vadis? Eur J Neurol 23, 437-438.

[26] Holtzer R, Epstein N, Mahoney JR, Izzetoglu M, Blumen HM (2014) Neuroimaging of mobility in aging: A targeted review. J Gerontol A Biol Sci Med Sci 69, 1375-1388.

[27] Posner HB, Tang MX, Luchsinger J, Lantigua R, Stern Y, Mayeux R (2002) The relationship of hypertension in the elderly to $\mathrm{AD}$, vascular dementia, and cognitive function. Neurology 58, 1175-1181.

[28] Oveisgharan S, Hachinski V (2010) Hypertension, executive dysfunction, and progression to dementia: The canadian study of health and aging. Arch Neurol 67, 187-192.

[29] Hajjar I, Quach L, Yang F, Chaves PH, Newman AB, Mukamal K, Longstreth W Jr, Inzitari M, Lipsitz LA (2011) Hypertension, white matter hyperintensities, and concurrent impairments in mobility, cognition, and mood: The Cardiovascular Health Study. Circulation 123, 858-865.

[30] Montero-Odasso M, Annweiler C, Hachinski V, Islam A, Toma N, Vasudev A (2012) Vascular burden predicts gait, mood, and executive function disturbances in older adults with mild cognitive impairment: Results from the gait and brain study. J Am Geriatr Soc 60, 1988-1990.

[31] Rosano C, Brach J, Longstreth WT Jr, Newman AB (2006) Quantitative measures of gait characteristics indicate prevalence of underlying subclinical structural brain abnormalities in high-functioning older adults. Neuroepidemiology 26, 52-60.

[32] Hausdorff JM, Yogev G, Springer S, Simon ES, Giladi N (2005) Walking is more like catching than tapping: Gait in the elderly as a complex cognitive task. Exp Brain Res 164, 541-548.

[33] Yogev-Seligmann G, Hausdorff JM, Giladi N (2008) The role of executive function and attention in gait. Mov Disord 23, 329-342.

[34] Lundin-Olsson L, Nyberg L, Gustafson Y (1997) "Stops walking when talking" as a predictor of falls in elderly people. Lancet 349,617 .

[35] Woollacott M, Shumway-Cook A (2002) Attention and the control of posture and gait: A review of an emerging area of research. Gait Posture 16, 1-14.

[36] Beauchet O, Dubost V, Gonthier R, Kressig RW (2005) Dual-task-related gait changes in transitionally frail older adults: The type of the walking-associated cognitive task matters. Gerontology 51, 48-52.

[37] Ble A, Volpato S, Zuliani G, Guralnik JM, Bandinelli S, Lauretani F, Bartali B, Maraldi C, Fellin R, Ferrucci L (2005) Executive function correlates with walking speed in older persons: The InCHIANTI study. J Am Geriatr Soc 53, 410-415.

[38] Bootsma-van der Wiel A, Gussekloo J, de Craen AJ, van Exel E, Bloem BR, Westendorp RG (2003) Walking and talking as predictors of falls in the general population: The Leiden 85-Plus Study. J Am Geriatr Soc 51, 1466-1471.

[39] Camicioli R, Howieson D, Lehman S, Kaye J (1997) Talking while walking: The effect of a dual task in aging and Alzheimer's disease. Neurology 48, 955-958.

[40] Camicioli R, Bouchard T, Licis L (2006) Dual-tasks and walking fast: Relationship to extra-pyramidal signs in advanced Alzheimer disease. J Neurol Sci 248, 205-209.

[41] Petersen RC, Doody R, Kurz A, Mohs RC, Morris JC, Rabins PV, Ritchie K, Rossor M, Thal L, Winblad B (2001) Current concepts in mild cognitive impairment. Arch Neurol 58, 1985-1992.

[42] Petersen RC (2004) Mild cognitive impairment as a diagnostic entity. J Intern Med 256, 183-194.

[43] Ritchie K, Touchon J (2000) Mild cognitive impairment: Conceptual basis and current nosological status. Lancet $\mathbf{3 5 5}$, 225-228.

[44] Montero-Odasso M, Bergman H, Phillips NA, Wong C, Sourial N, Chertkow H (2006) The effect of executive and memory dysfunction in gait performance in a cognitive impairment population. J Am Geriatric Soc 54, S154. 
[45] Bloem BR, Valkenburg VV, Slabbekoorn M, Willemsen MD (2001) The Multiple Tasks Test: Development and normal strategies. Gait Posture 14, 191-202.

[46] Hausdorff JM, Cudkowicz ME, Firtion R, Wei JY, Goldberger AL (1998) Gait variability and basal ganglia disorders: Stride-to-stride variations of gait cycle timing in Parkinson's disease and Huntington's disease. Mov Disord 13, 428-437.

[47] Hausdorff JM, Balash J, Giladi N (2003) Effects of cognitive challenge on gait variability in patients with Parkinson's disease. J Geriatr Psychiatry Neurol 16, 53-58.

[48] Holmes JD, Jenkins ME, Johnson AM, Adams SG, Spaulding SJ (2010) Dual-task interference: The effects of verbal cognitive tasks on upright postural stability in Parkinson's disease. Parkinsons Dis 2010, 696492.

[49] Morris M, Iansek R, Smithson F, Huxham F (2000) Postural instability in Parkinson's disease: A comparison with and without a concurrent task. Gait Posture 12, 205-216.

[50] Yogev G, Giladi N, Peretz C, Springer S, Simon ES, Hausdorff JM (2005) Dual tasking, gait rhythmicity, and Parkinson's disease: Which aspects of gait are attention demanding? Eur J Neurosci 22, 1248-1256.

[51] Allali G, Kressig RW, Assal F, Herrmann FR, Dubost V, Beauchet O (2007) Changes in gait while backward counting in demented older adults with frontal lobe dysfunction. Gait Posture 26, 572-576.

[52] Allali G, Dubois B, Assal F, Lallart E, de Souza LC, Bertoux M, Annweiler C, Herrmann FR, Levy R, Beauchet O (2010) Frontotemporal dementia: Pathology of gait? Mov Disord 25, 731-737.

[53] Rucco R, Agosti V, Jacini F, Sorrentino P, Varriale P, De SM, Milan G, Montella P, Sorrentino G (2017) Spatio-temporal and kinematic gait analysis in patients with Frontotemporal dementia and Alzheimer's disease through 3D motion capture. Gait Posture 52, 312-317.

[54] Hausdorff JM (2005) Gait variability: Methods, modeling and meaning. J Neuroeng Rehabil 2, 19.

[55] Herman T, Giladi N, Gurevich T, Hausdorff JM (2005) Gait instability and fractal dynamics of older adults with a "cautious" gait: Why do certain older adults walk fearfully? Gait Posture 21, 178-185.

[56] Paleacu D, Shutzman A, Giladi N, Herman T, Simon ES, Hausdorff JM (2007) Effects of pharmacological therapy on gait and cognitive function in depressed patients. Clin Neuropharmacol 30, 63-71.

[57] von EE, Altman DG, Egger M, Pocock SJ, Gotzsche PC, Vandenbroucke JP (2007) The Strengthening the Reporting of Observational Studies in Epidemiology (STROBE) statement: Guidelines for reporting observational studies. Lancet 370, 1453-1457.

[58] Farhan SM, Bartha R, Black SE, Corbett D, Finger E, Freedman M, Greenberg B, Grimes DA, Hegele RA, Hudson C, Kleinstiver PW, Lang AE, Masellis M, McIlroy WE, McLaughlin PM, Montero-Odasso M, Munoz DG, Munoz DP, Strother S, Swartz RH, Symons S, Tartaglia MC, Zinman L, Investigators ONDRI, Strong MJ (2017) The Ontario Neurodegenerative Disease Research Initiative (ONDRI). Cdn J Neurol Sci 44, 196-202.

[59] Nasreddine ZS, Phillips NA, Bedirian V, Charbonneau $\mathrm{S}$, Whitehead V, Collin I, Cummings JL, Chertkow H (2005) The Montreal Cognitive Assessment, MoCA: A brief screening tool for mild cognitive impairment. J Am Geriatr Soc 53, 695-699.

[60] Fazekas F, Chawluk JB, Alavi A, Hurtig HI, Zimmerman RA (1987) MR signal abnormalities at 1.5 T in Alzheimer's dementia and normal aging. AJR Am J Roentgenol 149, 351356.

[61] Dade LA, Gao FQ, Kovacevic N, Roy P, Rockel C, O'Toole CM, Lobaugh NJ, Feinstein A, Levine B, Black SE (2004) Semiautomatic brain region extraction: A method of parcellating brain regions from structural magnetic resonance images. Neuroimage 22, 1492-1502.

[62] Hittmair-Delazer M, Semenza C, Denes G (1994) Concepts and facts in calculation. Brain 117(Pt 4), 715-728.

[63] Verghese J, Kuslansky G, Holtzer R, Katz M, Xue X, Buschke H, Pahor M (2007) Walking while talking: Effect of task prioritization in the elderly. Arch Phys Med Rehabil 88, 50-53.

[64] Powell LE, Myers AM (1995) The Activities-specific Balance Confidence (ABC) Scale. J Gerontol A Biol Sci Med Sci 50A, M28-M34.

[65] Myers AM, Powell LE, Maki BE, Holliday PJ, Brawley LR, Sherk W (1996) Psychological indicators of balance confidence: Relationship to actual and perceived abilities. J Gerontol A Biol Sci Med Sci 51, M37-M43.

[66] Tinetti ME, Baker DI, McAvay G, Claus EB, Garrett P, Gottschalk M, Koch ML, Trainor K, Horwitz RI (1994) A multifactorial intervention to reduce the risk of falling among elderly people living in the community. $N$ Engl $J$ Med 331, 821-827.

[67] Muir SW, Berg K, Chesworth B, Speechley M (2008) Use of the Berg Balance Scale for predicting multiple falls in community-dwelling elderly people: A prospective study. Phys Ther 88, 449-459.

[68] Speechley M, Belfry S, Borrie MJ, Jenkyn KB, Crilly R, Gill DP, McLean S, Stolee P, Vandervoort AA, Jones GR (2005) Risk factors for falling among community-dwelling veterans and their caregivers. Can J Aging 24, 261-274.

[69] Liu T, Heitjan DF (2012) Sensitivity of the discrete-time Kaplan-Meier estimate to nonignorable censoring: Application in a clinical trial. Stat Med 31, 2998-3010.

[70] Abd ES, Torino C, D'Arrigo G, Bolignano D, Provenzano F, Mattace-Raso F, Zoccali C, Tripepi G (2012) An overview on standard statistical methods for assessing exposure-outcome link in survival analysis (Part II): The Kaplan-Meier analysis and the Cox regression method. Aging Clin Exp Res 24, 203-206.

[71] Cox TF, Czanner G (2016) A practical divergence measure for survival distributions that can be estimated from KaplanMeier curves. Stat Med 35, 2406-2421.

[72] Gill DP, Zou GY, Jones GR, Speechley M (2009) Comparison of regression models for the analysis of fall risk factors in older veterans. Ann Epidemiol 19, 523-530.

[73] Hochberg Y, Sharper A (1988) Bonferroni procedure for multiple tests of significance. Biometrika 75, 800-8002.

[74] Fritz S, Lusardi M (2009) White paper: "walking speed: The sixth vital sign". J Geriatr Phys Ther 32, 46-49.

[75] Middleton A, Fritz SL, Lusardi M (2015) Walking speed: The functional vital sign. J Aging Phys Act 23, 314-322.

[76] Montero-Odasso M, Schapira M, Soriano ER, Varela M, Kaplan R, Camera LA, Mayorga LM (2005) Gait velocity as a single predictor of adverse events in healthy seniors aged 75 years and older. J Gerontol A Biol Sci Med Sci 60, 1304-1309.

[77] Montero-Odasso M (2006) The value of gait velocity test for high-function populations. J Am Geriatr Soc 54, 1949-1950.

[78] Lord S, Howe T, Greenland J, Simpson L, Rochester L (2011) Gait variability in older adults: A structured review of testing protocol and clinimetric properties. Gait Posture 34, 443-450. 